\title{
Life Style Patterns and its Association with Menstrual Problems among Saudi Students in Eastern Province
}

\author{
Najwa K. Karout, PhD, MsM \\ Nursing Program, Ibn Sina National College for Medical Sciences \\ Jeddah, Saudi Arabia
}

\section{Correspondence}

Dr. Najwa K. Karout

P.O. Box 40997, Jeddah 21511, Saudi Arabia

e.M:nkkarout@yahoo.com

Submission: 12 May 2015

Accepted: $\quad 10$ Sep. 2015

\section{Citation}

Karout NK. Life style patterns and its association with menstrual problems among Saudi students in Eastern Province. JKAU Med Sci 20xx; 22 (4): 19-26. DOI: 10.4197/Med. 22.4 .3

\begin{abstract}
This paper aims to evaluate the lifestyle of young Saudi women and to examine any association between eating habits, exercise and smoking tobacco with different menstrual dysfunctions. A cross-sectional/ correlational survey was conducted on a sample of 412 students from the nursing college in Al Khobar in the Eastern province. The data was collected using a self-administered questionnaire relating to menstrual problems and lifestyle patterns and revealed that an unhealthy lifestyle was common among young Saudi women and was a major concern for public health specialists. Analysis of the data also showed that eating fast food increased the risk of premenstrual syndrome, dysmenorrhea and amenorrhea. Smoking had a significant association with premenstrual syndrome and hypermenorrhea; skipping breakfast had an inverse association with amenorrhea. However, the current study did not find any significant association between doing exercise and menstrual disorders. In conclusion, a new trend of lifestyle in Saudi culture needs to be assessed deeply, programs for health promotion are highly recommended, and additional related studies are needed.
\end{abstract}

\section{Keywords}

Life style; Skipping breakfast; Menstrual problem; Eating habits; Exercise; Smoking

\section{Introduction}

A healthy lifestyle is a valuable resource for reducing the incidence of diseases. Research shows that there is vast scientific evidence emphasizing the importance of lifestyle in achieving a healthy society. Eating habits, exercising and smoking, as well as the consumption of alcohol and drugs are important factors responsible for an individual's lifestyle ${ }^{[1]}$. Following a healthy lifestyle practice has been proven to reduce the occurrence of diseases and mortality

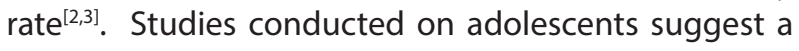

strong link between unhealthy habits and their overall health ${ }^{[4]}$.

Adopted habits in the early stages of life affect the risk of diseases later in life and fortunately, many of these factors are avoidable if identified ${ }^{[5]}$. One of the recent studies has shown an increased awareness among university students of healthy behaviors. Factors considered for this study were behavioral health risks such as tobacco use, alcohol and substance abuse, and improper diet and physical activities ${ }^{[6,7]}$. Changing diet patterns, like the intake of high/low calorie food, 
ingestion of micronutrients/difficulties in absorption, unsaturated fat and fiber consumption as well as increasing environmental toxins, not only influence the present lifestyle, but also induce gynecologic disorders such as dysmenorrhea and irregular menstruation. One study has shown that skipping breakfast is associated with menstrual disorders too ${ }^{[8]}$.

Cigarette smoking is one of the influential factors on reproduction, which causes delayed conception, increased miscarriage risk, shorter and irregular menstrual cycles, lower ovarian follicle density, increased dysmenorrhea, as well as the early onset of menopause. Cigarette smoking may also influence thyroid function ${ }^{[9]}$. Previous studies have shown that cigarette smoking causes decreased duration of bleeding, increased amount of bleeding per day and increased duration of dysmenorrhea ${ }^{[10,11]}$. On the other hand, engaging in regular exercise is a lifestyle pattern that positively influences the symptoms of premenstrual syndrome (PMS) ${ }^{[12]}$, whereas overexercising, usually seen among athletes, can have a negative impact ${ }^{[13]}$.

Irregular menstruation may act as a lead for the early detection of the hypothalamic-pituitary-ovarian axis dysfunction ${ }^{[14]}$ and can be defined by having an interval that is less than 23 days (polymenorrhea) or more than 32 days (oligomenorrhea), with a duration that is less than 3 days (hypomenorrhea) or more than 7 days (hypermenorrhea), missing menses for more than 3 months (amenorrhea) and having an abnormal amount of blood ${ }^{[15]}$. In addition, PMS is a multi-factorial syndrome that affects adolescent girls and is characterized as a psychological disorder and/or somatic complaint. These symptoms are recurrent, and they rapidly disappear just after the onset of menstruation ${ }^{[16]}$. In Saudi Arabia, studies have shown a high prevalence of sedentary behavior, physical inactivity, smoking and unhealthy dietary habits among adolescents, which has become a major concern for public health specialists ${ }^{[17]}$.

There is an urgent need for national policies to promote a healthy lifestyle among adults in the early stages of life in Saudi Arabia, particularly women. Therefore, it is important to evaluate the present lifestyle situation among Saudi women and estimate its influence on their menstrual cycles particularly because it affects the risk of diseases later in life.

The purpose of the current study is to evaluate the lifestyles of Saudi women during their early stages of life and to assess the presence of any association between eating habits, exercising and smoking tobacco, and different menstrual dysfunctions.

\section{Materials and Methods}

In the current study, a cross-sectional survey was conducted in a female nursing college in Al Khobar in the Eastern Province of Saudi Arabia. The sample size was estimated using Epi Info 6.04d, with a confidence (1-a) of $95 \%$, anticipated prevalence of $27 \%$ and margin of error of \pm 5 . The minimum sample size calculated was 280 (prevalence $27 \%$ of irregular menstruation among women ${ }^{[18]}$, to tolerate with non- responses. All 412 female students in the college were selected for the sample; these students registered in the academic year 2013-2014 and came from different areas of the province.

The data was collected using a structured, selfadministered, anonymous questionnaire that was designed in English and that included the following sections: demographic data, life style patterns, and menstrual problems.

The questionnaire prepared was based on previous similar studies for menstrual disorders and lifestyle

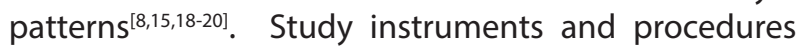
were pre-tested in the field and modified accordingly.

After taking the approval of the ethical committee of the college and under the permission of college authority, the questionnaire was delivered to all students in four academic years during a period of four days based on their academic schedule, taking into consideration the ethical issues. The purpose, design, benefits, and the method of study were explained by the researcher, also the way of completing the answers related to menstrual problems and lifestyle patterns, were clarified. The students were informed that participation was optional and not compulsory. An informed consent was attached to the questionnaire. It emphasized that all data collected would be strictly confidential and it mentioned that there was no need to write the name of the participants. Students were asked to feel free to complete the questionnaire within 30 minutes and hand it back to the researcher. Empty boxes were placed in the classes for this reason.

The researcher explained the operational definition of the lifestyle variables included in the questionnaire to the participants as follows:

Skipping breakfast: Not having the first meal (except coffee and water) before noon at least 5 times per week ${ }^{[21]}$. 
Doing exercise: Any regular physical activity at least three times a week for a minimum of 20 minutes ${ }^{[22]}$. Physical activity includes running or jogging, cycling, housework or yard work, as well as sports involving physical movement e.g., jumping rope, climbing stairs, roller-skating, swimming, cricket, soccer, badminton, basketball, handball, etc., and the frequency of participation in physical activity is measured per week $^{[23]}$.

Eating fast food: when the consumption $\geq$ once a week of any kind of food like common menu items at fast food outlets, including fish and chips, sandwiches, hamburgers, fried chicken, French fries, chicken nuggets, tacos, pizza and hot dogs ${ }^{[23]}$.

Regular smoker: According to the National Institute for Health Education Risk Prevention (INPES), a regular smoker is somebody who admits to smoking at least one cigarette (or equivalent) per day ${ }^{[24]}$.

Menstrual problems are explained to students as any irregularity in the duration, interval and blood loss during menstruation, also having amenorrhea, dysmenorrhea and PMS.

The researcher claims that students would be considered to have amenorrhea if they have had a delay of menstruation for the last three months and were not pregnant or breastfeeding. However, students who have the following symptoms (painful tender breasts, bloating or swelling of the abdomen, rapid mood changes, depressed mood) 10 days before menstruation and that vanish with the start of menstruation would be considered to have PMS ${ }^{[15]}$.

The collected data was analyzed using SPSS Statistics for Windows, Version 17, (SPSS Inc., Chicago, IL USA). Data was revised, coded and tabulated using frequency and percentage. To analyze and interpret the results, logistic regression was used and the $p$ value was considered as $<0.05$.

\section{Results}

Out of the 412 Saudi students recruited for the study, 342 completed the questionnaire (83\%), whereas $9.2 \%$ were absent and $7.8 \%$ rendered incomplete questionnaires. The results are shown in the following Tables 1, 2, 3 and Chart 1.

In Table 1 the results show that the majority of students (86.8\%) were between $18-23$ years old and $81 \%$ were single. With regards to the academic level,
$80.4 \%$ were under the $3^{\text {rd }}$ year. In most cases $(52.9 \%)$ the mother's educational level was equal to or less than intermediate.

The results in Table 2 show a high prevalence of non-healthy lifestyles among nursing students: $42.2 \%$ of the participants skipped breakfast, and $36 \%$ ate fast food or junk food. Moreover, $4.4 \%$ of the participants smoked tobacco regularly, and only $11 \%$ practiced exercise.

The results in Chart 1 show that $2.9 \%$ had secondary amenorrhea, while $21.6 \%$ and $21.3 \%$ of the participants had oligo and polymenorrhea, respectively. Also $6.7 \%$ and $9.1 \%$ had hypomenorrhea and hypermenorrhea, respectively. In addition, $96.3 \%$ of the participants had dysmenorrhea and $52.7 \%$ of the participants had at least 3 premenstrual symptoms, and these symptoms disappeared by the onset of menstruation.

The results in Table 3 show that eating fast food increases the risk of PMS dysmenorrhea and amenorrhea. Smoking has a significant association with PMS and hypermenorrhea, skipping breakfast has

Table 1. Background characteristics of the sample of the students $(n=342)$.

\begin{tabular}{|l|c|c|}
\hline \multicolumn{1}{|c|}{ Variable } & Frequency & Percent (\%) \\
\hline Age & 23 & $6.72 \%$ \\
\hline$<18$ & 297 & $86.84 \%$ \\
\hline $18-23$ & 22 & $6.43 \%$ \\
\hline$>23$ & 277 & $80.99 \%$ \\
\hline Marital Status \\
\hline Single \\
\hline Married \\
\hline Academic Year \\
\hline Foundation - 3rdYear & 275 & $80.00 \%$ \\
\hline$>3^{\text {rd } Y e a r ~}$ \\
\hline Mother Education & 67 & $19.59 \%$ \\
\hline$<$ Intermediate & 182 & $53.21 \%$ \\
\hline > Intermediate & 160 & $46.78 \%$ \\
\hline \multicolumn{2}{|l}{} \\
\hline
\end{tabular}

Table 2. Life style patterns among the sample of nursing students $(n=342)$.

\begin{tabular}{|c|c|c|}
\hline Variable & Frequency & Percent (\%) \\
\hline \multicolumn{3}{|l|}{ Skipping Breakfast } \\
\hline Yes & 145 & $42.39 \%$ \\
\hline \multicolumn{3}{|l|}{ Eat Fast Food } \\
\hline Yes & 124 & $36.25 \%$ \\
\hline \multicolumn{3}{|c|}{ Smoke Tobacco Regularly } \\
\hline Yes & 15 & $4.38 \%$ \\
\hline \multicolumn{3}{|l|}{ Practicing Exercise } \\
\hline Yes & 38 & $11.11 \%$ \\
\hline
\end{tabular}


Table 3. Association between life style patterns and menstrual dysfunction among nursing students $(n=342)$.

\begin{tabular}{|c|c|c|c|c|c|c|}
\hline & \multirow{2}{*}{ B } & \multirow{2}{*}{ df } & \multirow{2}{*}{ Sig. } & \multirow{2}{*}{$\operatorname{Exp}(B)$} & \multicolumn{2}{|c|}{ 95\% Confidence Interval for $\operatorname{Exp(B)}$} \\
\hline & & & & & Upper Bound & Upper Bound \\
\hline \multicolumn{7}{|l|}{ Premenstrual Syndrome } \\
\hline Breakfast Skipping & -0.08 & 1 & 0.75 & 0.923 & 0.556 & 1.532 \\
\hline Eating Fast Food & 0.547 & 1 & 0.04 & 1.727 & 1.012 & 2.948 \\
\hline Smoking Tobacco & -0.819 & 1 & 0.04 & 0.441 & 0.202 & 0.962 \\
\hline Practicing Exercise & 0.3 & 1 & 0.23 & 1.35 & 0.824 & 2.211 \\
\hline \multicolumn{7}{|l|}{ Dysmenorrhea } \\
\hline Breakfast Skipping & -0.303 & 1 & 0.65 & 0.738 & 0.198 & 2.746 \\
\hline Eating Fast Food & 2.06 & 1 & 0.05 & 7.848 & 0.926 & 66.494 \\
\hline Smoking Tobacco & -0.347 & 1 & 0.72 & 0.707 & 0.106 & 4.703 \\
\hline Practicing Exercise & 0.674 & 1 & 0.35 & 1.962 & 0.474 & 8.126 \\
\hline \multicolumn{7}{|l|}{ Oligomenorrhea } \\
\hline Breakfast Skipping & 0.208 & 1 & 0.47 & 1.231 & 0.695 & 2.179 \\
\hline Eating Fast Food & 0.028 & 1 & 0.93 & 1.029 & 0.547 & 1.934 \\
\hline Smoking Tobacco & 0.578 & 1 & 0.2 & 1.783 & 0.729 & 4.359 \\
\hline Practicing Exercise & 0.493 & 1 & 0.08 & 1.638 & 0.931 & 2.881 \\
\hline \multicolumn{7}{|l|}{ Polymenorrhea } \\
\hline Breakfast Skipping & -.097 & 1 & 0.69 & 0.908 & 0.556 & 1.483 \\
\hline Eating Fast Food & 0.227 & 1 & 0.41 & 1.255 & 0.729 & 2.162 \\
\hline Smoking Tobacco & 0.205 & 1 & 0.60 & 1.227 & 0.56 & 2.688 \\
\hline Practicing Exercise & -.269 & 1 & 0.27 & 0.764 & 0.471 & 1.239 \\
\hline \multicolumn{7}{|l|}{ Hypomenorrhea } \\
\hline Breakfast Skipping & 1.123 & 1 & 0.09 & 3.073 & 0.823 & 11.476 \\
\hline Eating Fast Food & -.451 & 1 & 0.52 & 0.637 & 0.161 & 2.513 \\
\hline Smoking Tobacco & 1.205 & 1 & 0.2 & 3.335 & 0.523 & 21.26 \\
\hline Practicing Exercise & 0.675 & 1 & 0.27 & 1.963 & 0.583 & 6.606 \\
\hline \multicolumn{7}{|l|}{ Amenorrhea } \\
\hline Breakfast Skipping & -.785 & 1 & 0.01 & 0.456 & 0.238 & 0.873 \\
\hline Eating Fast Food & 0.674 & 1 & 0.05 & 1.963 & 0.977 & 3.943 \\
\hline Smoking Tobacco & -.824 & 1 & 0.11 & 0.439 & 0.157 & 1.226 \\
\hline Practicing Exercise & -.081 & 1 & 0.79 & 0.922 & 0.497 & 1.712 \\
\hline \multicolumn{7}{|l|}{ Hypermenorrhea } \\
\hline Breakfast Skipping & -.534 & 1 & 0.08 & 0.586 & 0.322 & 1.066 \\
\hline Eating Fast Food & -.311 & 1 & 0.33 & 0.733 & 0.392 & 1.371 \\
\hline Smoking Tobacco & -1.275 & 1 & 0.01 & 0.28 & 0.097 & 0.804 \\
\hline Practicing Exercise & 0.694 & 1 & 0.02 & 2.002 & 1.106 & 3.623 \\
\hline
\end{tabular}

Abbr: df: Degree of freedom; Sig: Significance; $\beta$ : Coefficient; Exp $\beta$ : Exponentiation of the B coefficient.

an inverse association with amenorrhea. The current study did not find any significant association between doing exercise and menstrual disorders.

\section{Discussion}

The Saudi Arabian society is largely traditional and Islamic. It values its culture and habits. The habits of the people here are clearly distinguishable from their counterparts in western societies in many respects. The pattern of the early adult's problems is expected to be different from that in the West ${ }^{[25]}$. As per the current study, the results showed that students in Saudi Arabia from the Eastern province also have similar unhealthy lifestyles to their colleagues in the world. Although they are nursing students and are supposed to know the importance of a healthy lifestyle, $42.2 \%$ of them skip breakfast and $36 \%$ eat fast food, which is almost comparable to the results found in other studies among Saudi students and other nationalities in schools and colleges $^{[8,17,18]}$. This could be an alarming trend about the new Saudi young adults' lifestyle. Furthermore, one study conducted about smoking habits shows that the prevalence of smoking among Saudi females in college is between $2.4-37 \%{ }^{[26]}$. Another study showed between $0.7-11 \%{ }^{[24]}$, which is similar to our study showing $4.4 \%$ 
Chart 1

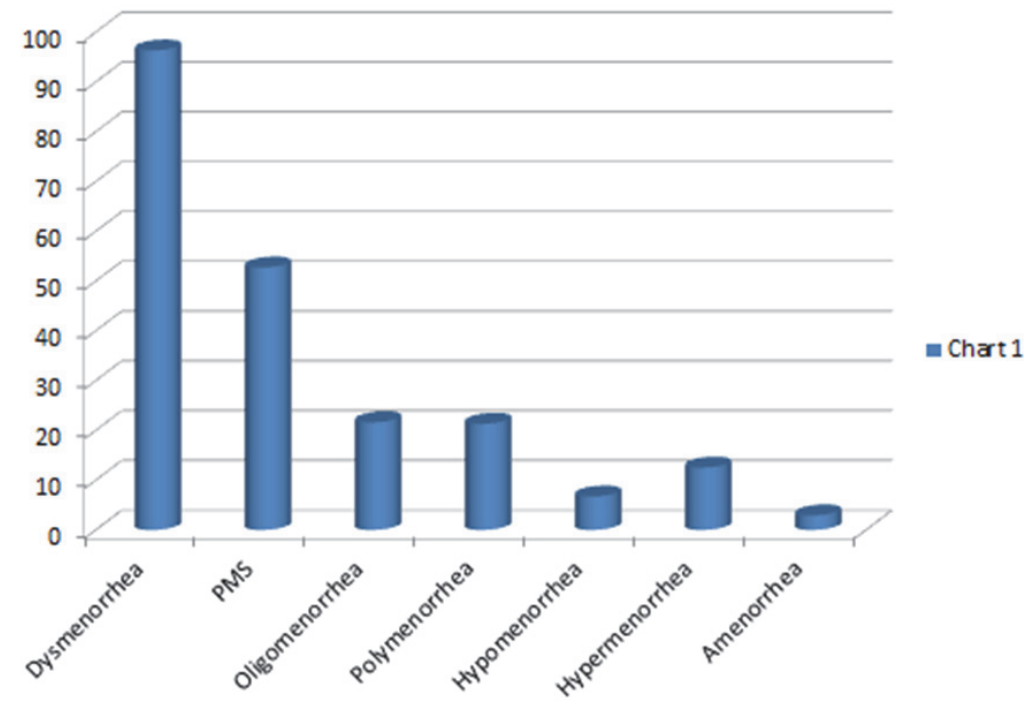

Chart 1. Menstrual cycle pattern among the sample of nursing students ( $n=342)$. (PMS: Premenstrual syndrome).

of the female students admitted regular tobacco use. We had a considerable percentage of females (65\%) showing occasional smoking habits, which is clearly on the higher side when compared to other studies. Therefore, the findings highlight the importance of implementation of tobacco cessation with the help of the community and non-governmental organizations.

According to one of the studies conducted on exercise habits among Saudi female students, around $20 \%$ claimed they practiced exercise regularly ${ }^{[17]}$, while another study found that $69.3 \%$ of Saudi females practice exercise only occasionally ${ }^{[27]}$. However, in our findings the results showed less prevalence of participating in physical exercises and workouts, with around $11 \%$ of the participants practicing regular physical exercise. Programs should be designed to encourage female adults in Saudi to take part in physical activity, although within the Saudi context and its special culture, it is difficult for females to arrange for such activities. Therefore, it is better to encourage that in the colleges and schools.

In the logistic regression analysis, eating fast food has a significant association with PMS, dysmenorrhea and amenorrhea. Consequently, in the current study, the results showed that those who ate fast food had a higher tendency to suffer from PMS; similar to the results found in one of the studies reported ${ }^{[20]}$. But unlike many studies published, we did not find any significant relationship between PMS and other lifestyle patterns (skipping breakfast, smoking and doing exercise $)^{[18,28,29]}$.

We have found a significant inverse association between skipping breakfast and amenorrhea as a protective risk factor. In another study conducted in Egypt, skipping breakfast had a strong relationship with dysmenorrhea, irregular menstruation, oligomenorrhea and premenstrual pain, which is different to the findings from our study ${ }^{[19]}$. Other studies have found that skipping breakfast had an impact on menstrual dysfunction, such as irregularity, dysmenorrhea, oligomenorrhea ${ }^{[8,19]}$. We did not find such relation similar to other studies ${ }^{[26]}$. This could be because of other unknown cofactors, so more indepth studies based on nutritional values of the high percentage of breakfast skipping habits need to be conducted, especially among Saudi students.

Despite how previous studies showed a significant association between doing exercise and menstrual dysfunctions ${ }^{[13,18,27,28]}$, the majority discussed the type of regular exercise and its effect on the duration and length of menstruation, PMS and dysmenorrhea. However, in the current study, no association was revealed, which could be according to the researcher's point of view that doing light regular exercise may help reduce anxiety, depression, stress responsivity, mood state, self- esteem, and body image ${ }^{[12,13]}$. 
In the logistic regression analysis of our study, smoking had a significant association with PMS and hypermenorrhea, and previous studies have shown similar results ${ }^{[20,30]}$.

Those who smoke tobacco regularly have a higher tendency to suffer from PMS and an increased length of menstruation, so national programs should be adopted for smoking cessation.

In our study, we found no significant association between lifestyle patterns and oligomenorrhea, polymenorrhea or hypomenorrhea, unlike some other studies. Lifestyle affects the quality of life as reported by many researchers and has an adverse effect on menstrual function. In light of the current study, we firmly believe that lifestyle does affect menstruation. However, a few studies have reported negative or no impact of lifestyle. Additional studies may be needed using a wider geographical scope and a larger sample.

Finally, the Saudi culture like other oriental cultures, values fertility, so preventing any risk factors such as menstrual problems by adopting a healthy lifestyle will help in this respect/area.

\section{Conclusion}

In summary, it is now obvious that there is a need for the evaluation of the present situation and the creation of awareness among college students of the effect of their lifestyle on the menstrual cycle. Lifestyle modifications, particularly promotion of the dietary and exercise habits, and cessation of smoking, must be emphasized in health-related initiatives of governmental and nongovernmental organizations.

\section{Conflict of Interest}

The author has no conflict of interest.

\section{Disclosure}

The author did not received any type of commercial support either in forms of compensation or financial for this study. The author has no financial interest in any of the products or devices, or drugs mentioned in this article.

\section{Ethical Approval}

Obtained.

\section{References}

[1] [No authors listed]. A healthy lifestyle. World Health Organization. Assessed on Sept. 2015. <http://www.euro. who.int/en/health-topics/disease-prevention/nutrition/ahealthy-lifestyle>.

[2] Nöthlings U, Ford ES, Kröger J, Boeing H. Lifestyle factors and mortality among adults with diabetes: Findings from the European Prospective Investigation into Cancer and Nutrition-Potsdam Study. J Diabetes 2010; 2(2): 112-117.

[3] Hu FB, Liu Y, Willett WC. Preventing chronic diseases by promoting healthy diet and lifestyle: public policy implications for China. Obes Rev 2011; 12(7): 552-559.

[4] Landsberg B, Plachta-Danielzik S, Lange D, Johannsen M, Seiberl J, Müller MJ. Clustering of lifestyle factors and association with overweight in adolescents of the Kiel Obesity Prevention Study. Public Health Nutr 2010; 13(10): 1708-1715.

[5] Gall SL, Jamrozik K, Blizzard L, Dwyer T, Venn A. Healthy lifestyles and cardiovascular risk profiles in young Australian adults: the Childhood Determinants of Adult Health Study. Eur J Cardiovasc Prev Rehabil 2009; 16(6): 684-689.

[6] Irwin JD. The prevalence of physical activity maintenance in a sample of university students: a longitudinal study. J AM Coll Health 2007; 56(1): 37-41.

[7] Laska MN, Pasch KE, Lust K, Story M, Ehlinger E. Latent class analysis of lifestyle characteristics and health risk behaviors among college youth. Prev Sci 2009; 10(4): 376-386.

[8] Fujiwara T, Nakata R. Skipping breakfast is associated with reproductive dysfunction in post-adolescent female college students. Appetite 2010; 55(3): 714-717.

[9] Whitcomb BW, Bodach SD, Mumford SL, Perkins NJ, Wactawski-Wende J, Liu A, Schisterman EF. Ovarian function and cigarette smoking. Paediatr Perinat Epidemiol 2010; 24(5): 433-440.

[10] Dorn LD, Negriff S, Huang B, Pabst S, Hillman J, Braverman, P. Menstrual symptoms in adolescent girls: association with smoking, depressive symptoms and anxiety. J Adolesc Health 2009; 44(3): 237-243.

[11] Liu Y, Gold EB, Lasley BL, Johnson WO. Factors affecting menstrual cycle characteristics. Am J Epidemiol 2004; 160(2): 131-140.

[12] Halbreich U. Algorithm for treatment of premenstrual syndromes (PMS): experts' recommendations and limitations. Gynecol Endocrinol 2005; 20(1): 48-56.

[13] Gudmundsdottir SL, Flanders WD, Augestad LB. A longitudinal study of physical activity and menstrual cycle characteristics in healthy Norwegian women - The NordTrøndelag Health Study. Norsk Epidemiologi 2011; 20(2): 163-171.

[14] Arrais RF, Dib SA. The hypothalamus-pituitary-ovary axis and type 1 diabetes mellitus: a mini review. Hum Reprod J 2005; $21(2): 327-337$. 
[15] Karout N, Hawai SM, Altuwaijri S. Prevalence and pattern of menstrual disorders among Lebanese nursing students. East Mediterr Health J 2012: 18(4): 346-352.

[16] Bakhshani NM, Aghashahi Z, Pour KL, Yaghmaei M. Relationship between symptoms of premenstrual syndrome (PMS) and quality of life (QOL) in the adolescents. Life Sci J 2013; 10(2s): 265-268.

[17] Al-Hazzaa HM, Abahussain NM, Al-Sobayl HI, Qahwaji DM, Musaiger AO. Physical activity, sedentary behaviors and dietary habits among Saudi adolescents relative to age, gender and region. Int J Behav Nutr Phys Act 2011; 8: 140149.

[18] Lakshmi AS. Impact of life style and dietary habits on menstrual cycle of college students. IJSR 2013; 4(4): 28452847.

[19] Eittah HA. Effect of breakfast skipping on young females' menstruation. Health Sci J 2014; 8(4): 469-484.

[20] Seedhom AE, Mohammed ES, Mahfouz EM. Life style factors associated with premenstrual syndrome among ElMinia University Students, Egypt. ISRN Public Health 2013; 2013: 1-6.

[21] Holtzman NS. To skip or not to skip? Varying definitions of breakfast skipping and associations with disordered eating, obesity and depression. Wesleyan Uni, Middletown, CT; 2010: 4. Thesis.

[22] [No authors listed]. The benefits of physical activity for health and well-being. C3 Collaborating for Health. 2011.

[23] Mushtaq MU, Gull S, Mushtaq K, Shahid U, Shad MA, Akram J. Dietary behaviors, physical activity and sedentary lifestyle associated with overweight and obesity, and their socio-demographic correlates, among Pakistani primary school children. Int J Behav Nutr Phys Act 2011; 8: 130.

[24] National Institute for Health Education Risk Prevention (INPES) http://www.insee.fr/en/methodes/default. asp?page=definitions/fumeurs-reguliers.htm

[25] Naeem Z. Medical and behavioral problems among Saudi adolescents. Int J Health Sci (Qassim) 2013; 7(2): V-VI.

[26] Bassiony M. Smoking in Saudi Arabia. Saudi Med J 2009; 30(7): 876-881.

[27] Al-Gelban KS. Dietary habits and exercises practices among the students of a Saudi Teachers'Training College. Saudi J 2008; 29(5): 754-759.

[28] Cobb KL, Bachrach LK, Greendale G, Marcus R, Neer RM, Nieves J, Sowers MF, Brown BW Jr, Gopalakrishnan G, Luetters C, Tanner HK, Ward B, Kelsey JL. Disordered eating, menstrual irregularity, and bone mineral density in female runners. Med Sci Sports Exerc 2003; 35(5): 711-719.

[29] Mandal R, Sarkar AP, Ghorai S. A study on premenstrual syndrome among adolescent girl students in an urban area of West Bengal. Int J Reprod Contracept Obstet Gynecol. 2015; 4(4): 1012-1015.
[30] Bertone-Johnson ER, Hankinson SE, Johnson SR, Manson JE. Cigarette smoking and the development of premenstrual syndrome. Am J Epidemiol 2008; 168(8): 938-945. 


\title{
اسلوب الحياة وانماطها وارتباطها بمشاكل الطمث بين الطالبات في المنطقة
}

$$
\text { الشرقية للسعودية }
$$

\author{
نجوى كامل قاروط

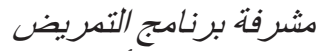

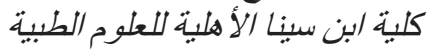

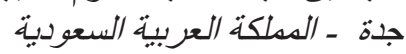

المستخأص. لتقيم أسلوب حياة الفتيات السعوديات ودراسة وجود أي علاقة بين عادات تناول الطعام، ممارسة التمارين

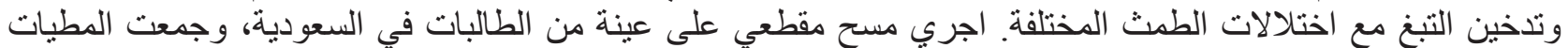

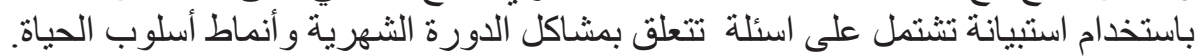

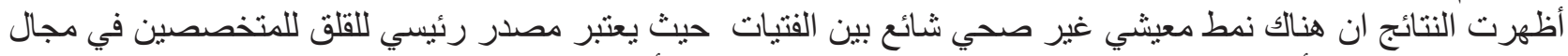

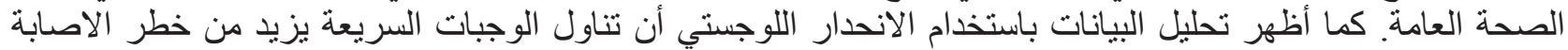

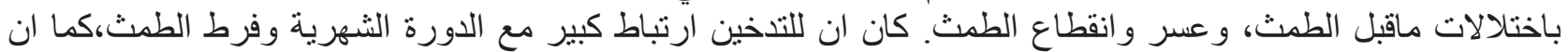

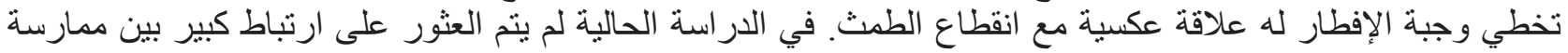

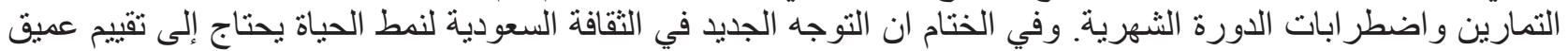

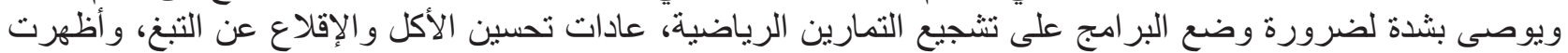

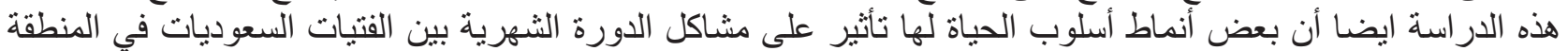

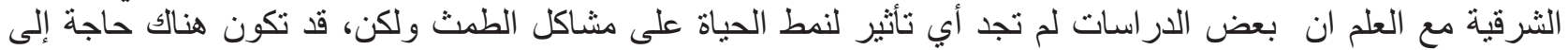

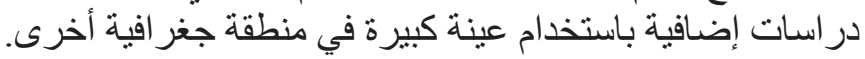

